\title{
Clinician Perspectives on the Benefits of Practice Facilita- tion for Small Primary Care Practices
}

\author{
Erin S. Rogers, DrPH ${ }^{1,2}$ \\ Allison M. Cutbel, MPH \\ Carolyn A. Berry, PbD ${ }^{1}$
}

Sue A. Kaplan, JD ${ }^{1}$

Donna R. Sbelley, MD, MPH ${ }^{1}$

'New York University School of Medicine, Department of Population Health, New York, New York

${ }^{2}$ VA NY Harbor Healthcare System, New York, New York
Conflicts of interest: authors report none.

\section{CORRESPONDING AUTHOR}

Erin S. Rogers, DrPH

New York University School of Medicine 180 Madison Ave

New York, NY 10016

Erin.Rogers@nyulangone.org

\begin{abstract}
PURPOSE Small independent primary care practices (SIPS) often lack the resources to implement system changes. HealthyHearts NYC, funded through the EvidenceNOW initiative of the Agency for Healthcare Research and Quality, studied the effectiveness of practice facilitation to improve cardiovascular diseaserelated care in 257 SIPs. We sought to understand SIP clinicians' perspectives on the benefits of practice facilitation.
\end{abstract}

METHODS We conducted in-depth interviews with 19 SIP clinicians enrolled in HealthyHearts NYC. Interviews were transcribed and coded using deductive and inductive approaches. To understand whether the perceived benefits of practice facilitation differ based on the availability of internal staff for quality improvement (QI), we compared themes pertaining to benefits between practices with 3 or fewer office staff vs more than 3 office staff.

RESULTS Clinicians perceived 2 main benefits of practice facilitation. First, facilitators served as a connection to the external health care environment for SIPS, often through teaching and information sharing. Second, facilitators provided electronic health record (EHR)/data expertise, often by teaching functionality and completing technical assistance and tasks. SIPs with more than 3 office staff felt that facilitators provided benefits primarily through teaching, whereas SIPs with 3 or fewer staff felt that facilitators also provided hands-on support. At the intersections of these benefits, there emerged 3 central practice facilitation benefits: (1) creating awareness of quality gaps, (2) connecting practices to information, resources, and strategies, and (3) optimizing the EHR for QI goals.

CONCLUSIONS SIP clinicians perceived practice facilitation to be an important resource for connecting their practice to the external health care environment and resources, and helping their practice build QI capacity through teaching, hands-on support, and EHR-driven solutions.

Ann Fam Med 2019;17:S17-S23. https://doi.org/10.1370/afm.2427.

\section{INTRODUCTION}

S mall independent primary care practices (SIPs), defined as those with 5 or fewer clinicians, provide primary care for a substantial proportion of the US population, ${ }^{1}$ yet the number of SIPs has been declining over the past 30 years. ${ }^{2}$ Health care policy changes and quality improvement (QI) requirements for incentive-based initiatives ${ }^{3,4}$ —for example, the Patient Centered Medical Home (PCMH), meaningful use, and advanced alternative payment models - have contributed to the reduction in SIPs, as these requirements put stressors on these practices having limited resources to implement the systems changes necessary to meet new quality standards. ${ }^{5,6}$

External practice facilitation can be an important strategy for enabling practice transformation in SIPs. ${ }^{7}$ External practice facilitators support practices with systems changes that range from distinct tasks (eg, chart auditing, electronic health record [EHR] template management) to more complex change processes (eg, team building, workflow redesign). ${ }^{8,9} \mathrm{~A}$ recent systematic review found that primary care practices with the support of a facilitator are almost 3 times more likely to implement evidencebased guidelines compared with usual care practices. ${ }^{7}$ 
SIP clinicians must perceive value in practice facilitation to want to enroll in, and allocate resources toward, a QI intervention offered by a practice facilitation organization..$^{10}$ Only a single article has assessed SIP clinician perspectives on the benefits of practice facilitation, ${ }^{11}$ thus limiting the field's ability to understand how this service affects SIP clinicians and the ability to design facilitation interventions to meet the needs of SIPs.

To fill this evidence gap, we conducted an analysis of interviews with SIP clinicians enrolled in a large randomized controlled trial testing a practice facilitation intervention. ${ }^{12}$ The analysis was designed to answer a primary research question: what are the perceived benefits of practice facilitation to SIP clinicians? Additionally, because the successful implementation of health care innovations often depends on the availability of technical assistance and resources, ${ }^{6}$ SIPs with very few internal support staff may benefit differentially from the resources offered through external practice facilitators. We therefore explored an additional secondary research question: do the perceived benefits of practice facilitation differ based on the number of internal, nonclinical staff available to a SIP?

\section{METHODS}

\section{Setting}

The study was approved by the New York University School of Medicine Institutional Review Board. Data came from interviews conducted with SIP clinicians enrolled in the parent trial, Healthy Hearts NYC (HHNYC). ${ }^{12}$ The HHNYC study used a steppedwedge cluster-randomized controlled trial design to evaluate the impact of practice facilitation on Million Hearts ABCS (aspirin, blood pressure, cholesterol, smoking cessation) outcomes in $257 \mathrm{SIPs}$ throughout New York City. ${ }^{12}$ Participating practices were randomly assigned to begin a multicomponent intervention during 1 of 4 study waves. The intervention aligned with the Chronic Care Model ${ }^{13,14}$ and consisted of 13 in-person visits by a practice facilitator employed by the Primary Care Information Project over 1 year..$^{15}$ The facilitators had completed the University of Buffalo's Practice Facilitator Certificate Program ${ }^{16}$ and in-house study training. At the beginning of the intervention, they reviewed with the practice their baseline ABCS outcome measures, and the practice decided in which order they would like to work each measure. Practice facilitators were responsible for helping practices improve their measures by implementing QI strategies such as EHR optimization (eg, running registries to identify high-risk patients), setting performance targets and goals, and providing performance feedback. ${ }^{12}$ In addition to data support, the facilitators were responsible for training clinicians and staff on evidence-based practices for addressing each ABCS measure, and assessing and redesigning office workflow. ${ }^{12}$

\section{Participants}

We used a purposeful sampling approach to identify and recruit interviewees to obtain diversity on 3 criteria: study wave, geographic region (the 5 New York City boroughs), and baseline ABCS performance (high, medium, or low) using a composite measure created for the study that assessed the proportion of patients in a practice with a history of atherosclerotic cardiovascular disease who met targets for 3 of the 4 Million Hearts outcomes (ABC, defined as receiving aspirin and statin therapy, and having their blood pressure controlled to $<140 / 90 \mathrm{~mm} \mathrm{Hg}) .{ }^{12,17} \mathrm{~A}$ member of the study team invited clinicians to participate in an interview via an institutional e-mail, with up to 3 telephone or in-person follow-up e-mails for nonresponders.

Figure 1 provides an overview of the recruitment process. A total of 87 clinicians were invited to participate in an interview. The final sample included 19 clinicians from 19 practices. We considered conducting additional interviewing but believed that thematic saturation had been achieved.

\section{Interview Procedures}

As part of the parent HHNYC study, investigators developed a broad, semistructured interview guide that asked about a range of issues affecting clinicians that was guided by the Consolidated Framework for Implementation Research. ${ }^{18}$ Questions included inquiries into the clinician's experience with the practice facilitation process. The guide was refined after the first 4 interviews, as investigators discussed field notes taken regarding the performance of certain questions.

Each clinician completed 1 interview. The interviews were conducted by 4 faculty investigators (E.S.R., C.A.B., S.A.K., D.R.S.) with qualitative interviewing experience. E.S.R., C.A.B., and S.A.K. had no prior relationship with interviewees. D.R.S. was the study principal investigator but had not interacted with practices before the interviews. Participants were told that the purpose of the interviews was to get a deeper understanding of their practice and their experience with the HHNYC study. Interviews were conducted from December 2016 through March 2018. The interviews were timed to take place at least 6 months after the practice facilitation intervention began at participating sites. Interviews ranged from 45 to 60 minutes and took place in the clinicians' private practice offices. Interviewers obtained verbal informed consent from all participants. Clinicians were paid $\$ 50$ 
for completing an interview. Interviews were audiotaped and transcribed verbatim.

\section{Data Analysis}

Analyses were conducted with Atlas.ti v. 8.1 (ATLAS. ti Scientific Software Development GmbH). Although the main HHNYC study's interviews covered a range of topics, the current analysis focused on interview content discussing the benefits of practice facilitation. Two investigators (E.S.R., A.M.C.) used both deductive and inductive (grounded theory) approaches to code the interviews. They created an initial codebook that included domains from the interview guide, domain/code definitions, and inclusion and exclusion criteria. They then independently coded 5 transcripts, discussed coding agreement and disagreement, and updated the codebook as needed. One researcher (A.M.C.) coded the remaining 13 transcripts, with co-coding of every fifth transcript. Once coding was complete, the 2 researchers met to identify the most common benefits endorsed (based on memos and the frequency of code occurrence in the qualitative data set) and to identify higher-level code relationships that became themes. To understand whether the perceived practice facilitation benefits differed based on a practice's number of internal support staff available for QI, the analysis was conducted separately for 11 practices that were at or below the sample's median number of nonclinical office staff ( 3 staff members) and 8 practices that were above this median.

\section{RESULTS}

\section{Participant Characteristics}

Table 1 shows the characteristics of participating clinicians and their practices and patients, stratified by office staff size. All practices were clinician owned. Most clinicians were male, were physicians, and had been at their practice approximately 15 to 17 years. Most practices with 3 or fewer office staff were soloclinician practices, whereas larger practices had additional full-time equivalent clinicians. Most practices had PCMH recognition and served predominantly nonwhite patients, with about one-third of patients having Medicaid.

\section{Themes Regarding Benefits of Practice Facilitation}

Figure 2 displays the perceived benefits themes and the roles that practice facilitators played in the practices. The figure depicts the 2 main interacting benefits of practice facilitation, external connection to the health

\section{Figure 1. Interview recruitment diagram.}

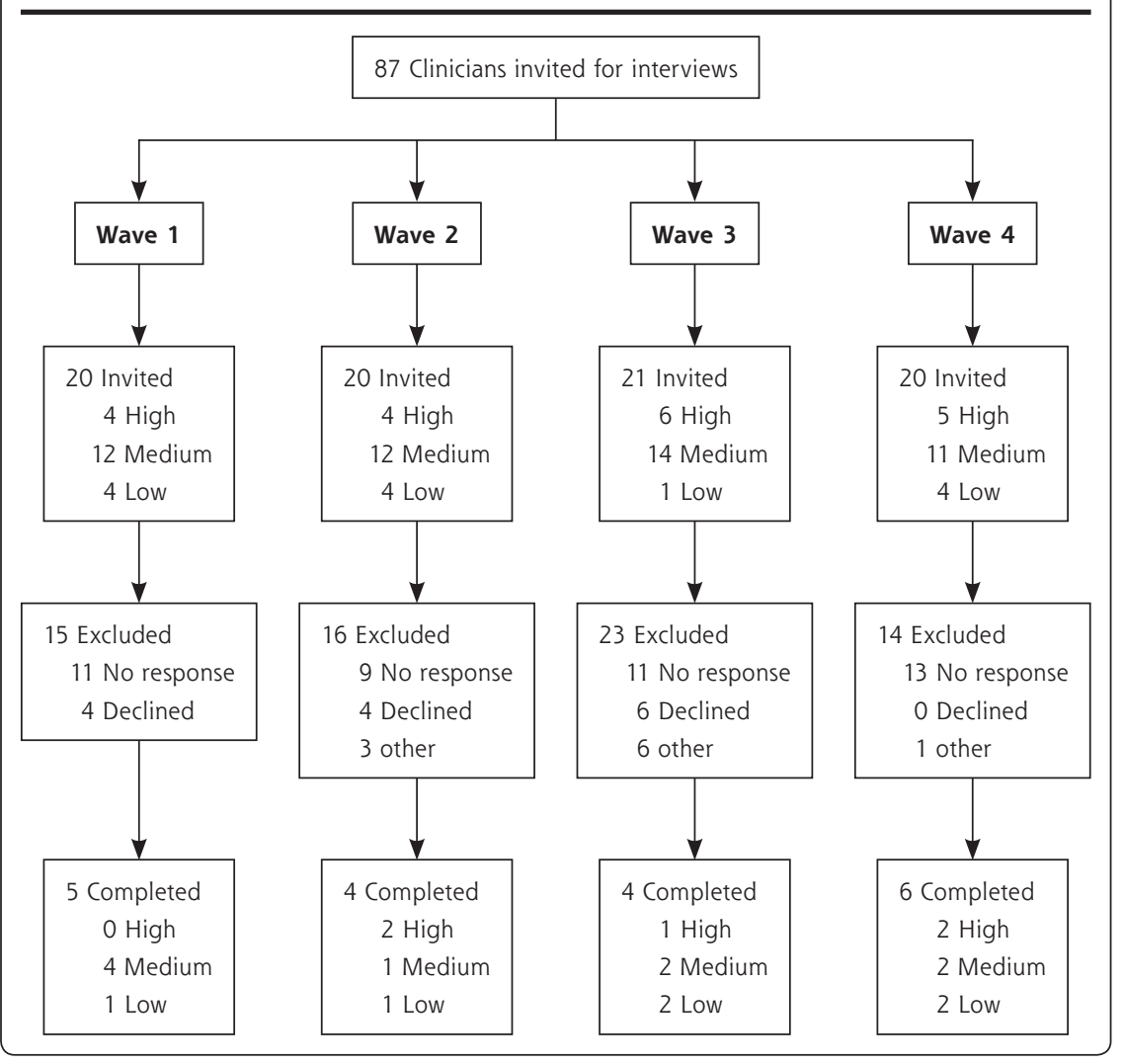

care system and EHR expertise, as well as the main perceived roles of practice facilitators, teachers, and support staff. At the intersection of the main benefits and roles, there emerged 3 centralized benefits. As indicated in the figure, certain benefits were solely or more frequently mentioned by the very small practices.

\section{Main Themes}

Connection to the External Health Care Environment. Clinicians in practice of both sizes believed that their practice facilitator connected their practice to the external health care environment. This external connection included teaching the practice through information sharing. Clinicians valued information given by their facilitator about external seminars to help improve their practice and information about new reporting require- 
ments and deadlines, as well new reimbursement standards and practice transformation models they were expected to participate in. One clinician noted, "All this new stuff that's coming out, like MACRA [Medicare Access and CHIP Reauthorization Act of 2015], I have no information about it. [Our practice facilitator] is basically our point of source of information. Anytime we have questions, we just ask her." (clinician 12 ; more than 3 staff)

Clinicians also valued information shared by their practice facilitator about patient resources and pro-

\section{Table 1. Characteristics of Participating Clinicians and Their Practices and Patients}

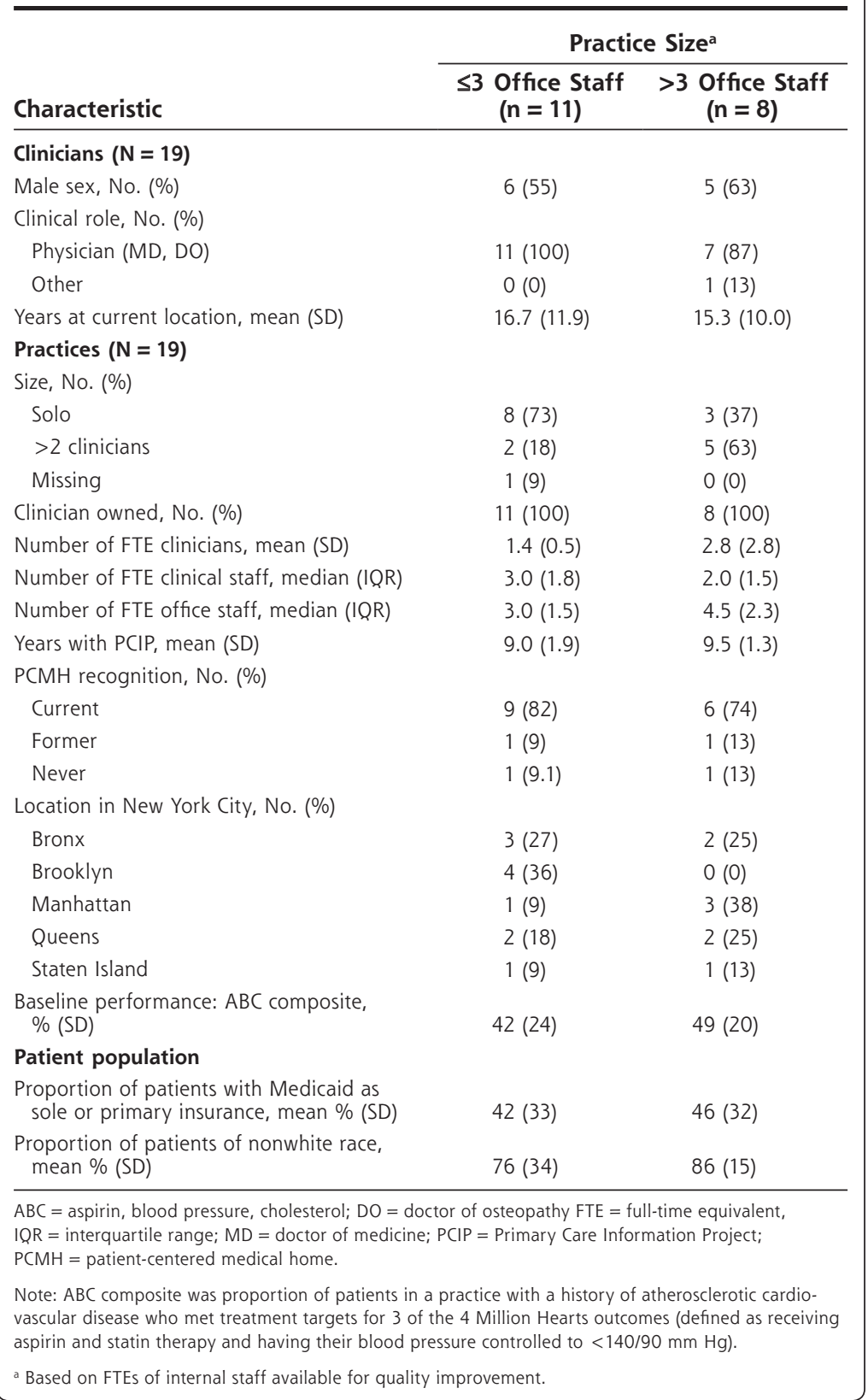

grams that clinicians would otherwise need to search for themselves. Clinicians especially benefited from information that targeted the needs of their patient population, for example, if their practice had a large burden of diabetes: "By far, I think the largest scourge we have out here for my patients is diabetes. One of the biggest factors attributing to cardiovascular disease is diabetes. [The practice facilitator] really helped us a lot and made me understand the National Diabetes Prevention Program." (clinician 19; more than 3 staff) As independent practices, the SIPs were often functioning in isolation, and clinicians did not always have a network of colleagues to share with and learn from. Interviews revealed that practice facilitators were able to connect different practices through sharing information and strategies that they learned through their work with multiple practices. One physician in a very small practice noted: "I appreciate [the practice facilitator's] insight because he sees other practices and he has ways that he can give us information that we would not otherwise get from other practices." (clinician $10 ; 3$ or fewer staff)

Lastly, clinicians valued information sharing through the dissemination of new clinical practice guidelines by the practice facilitators. This dissemination oriented clinicians to updated practice recommendations of which they were not always aware: "[The practice facilitator] is explaining to me some of the newer guidelines with blood pressure and even providing me with those ... the newer parameters, which I thought I knew, but I think there was some changes that I was not aware of." (clinician 14; 3 or fewer staff)

Practices with 3 or fewer office staff discussed 2 additional external connection benefits that occurred through hands-on support, rather than through teaching. Clinicians in these very small practices noted that their practice facilitator provided hands-on support in helping the practice complete their PCMH application or attest for meaningful use by ensuring data reporting was accurate for clinical quality measures. "[The 
practice facilitator] helped me with [Medicare's meaningful use]. If he wasn't helping, I don't think I would ever be able to do it on my own." (clinician $01 ; 3$ or fewer staff)

Lastly, clinicians in the very small practices were more likely to describe the value of the hands-on support they received from their practice facilitators when they were willing to serve as a liaison between the practice and external vendors or organizations. This pattern was most prominent when it came to the facilitator talking with EHR vendors to resolve operating issues or data issues that interfered with practice workflow, reimbursement, or implementation of the EHR-driven practice changes related to the HHNYC intervention, or some combination thereof. One physician described how his practice facilitator would "speak to the [EHR vendor] himself" (clinician 10) to resolve issues with the health record.

Provision of EHR/Data Expertise. The second main benefit described by clinicians of both practice sizes was the practice facilitators' EHR expertise and use of data. As with the external connection role, the facilitators provided their EHR/data expertise through both teaching and hands-on support. On the teaching side, clinicians believed that their practices benefited greatly from learning EHR functions that they either did not know about or did not know how to run, such as using clinical decision support systems, generating patient registries to identify high-risk patients, and using chronic condition templates for proper documentation of services related to HHNYC: "The [medical record] system is very powerful and we only know very little, just enough to operate. Each time [the practice facilitator] came, I learned more ... it's good to have someone here to introduce more functions to us. It's always good face-to-face learning." (clinician 08; more than 3 staff)

Some clinicians in both practice sizes (although more commonly those in the very small practices) noted that the practice facilitator's EHR/data expertise also included hands-on support. The facilitators provided this support through EHR technical assistance and by setting up and running EHR functions themselves for the practice during intervention visits (in addition to, or rather than, teaching the practice staff how to do this): "[The practice facilitator] was very instrumental in setting up parameters for us in the EHR. Any time we had any questions, she was always ready to either come or to guide us in the path to follow." (clinician 16; 3 or fewer staff)

\section{Central Themes}

At the intersections of the 2 main benefits described above (indicated by the central oval in Figure 2), there emerged 3 centralized practice facilitation benefits experienced by practices of all sizes: creating awareness of quality gaps; connecting the practice to information, resources, and strategies; and optimizing the EHR for QI goals.

Through the sharing of - and teaching aboutboth external information (eg, clinical practice guidelines) and EHR-derived internal information (eg, practice performance data), practice facilitators drew clinicians' attention to their own gaps in care and oriented the practices to pay attention to, and focus on, the highest-priority patients and care processes. Practice facilitators then used teaching and hands-on support to help connect the practices to both external and internal resources and strategies to help address the identified practice's needs (often through the optimization of the EHR).

\section{DISCUSSION}

\section{Key Findings}

Our interviews showed that SIP clinicians held positive views toward practice facilitation, thus supporting the continued development, testing, and funding of practice facilitation programs, and the recruitment of SIPs in particular into practice facilitation interventions. In

\section{Figure 2. Themes related to the perceived benefits and roles of external practice facilitation for small independent primary care practices.}

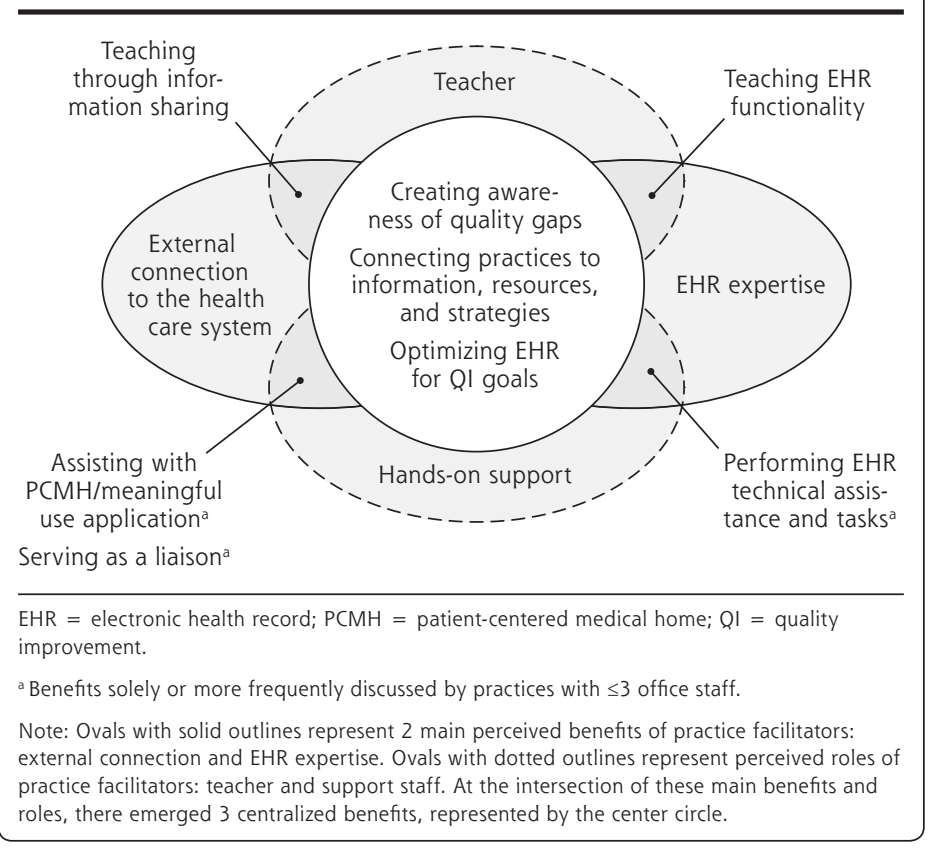


addition, consistent with prior literature, ${ }^{11,19}$ SIP clinicians having varying staff levels held similar perceptions of the overall benefits of practice facilitation by viewing their facilitators as an important connection to the external health care environment (eg, through information sharing, consistent with the Cooperative Extension Service model for the country's primary care system recommended by Grumbach and $\mathrm{Mold}^{20}$ ) and as an essential source of EHR expertise, which helped practices attend to quality gaps. Clinicians rarely mentioned other elements of the intervention (eg, workflow redesign) as benefits. They may not see the value of these suggested changes, or perhaps just view them to be not as important as the other elements they raised. Practice facilitation is a resource-intense intervention for practices and their facilitators. Future studies should seek to understand which elements are most important and effective to both engage and retain practices in practice facilitation and to motivate practices to adopt systems changes.

Although practices with 3 or fewer staff and practices with more than 3 staff held similar views on the benefits of practice facilitators, they differed somewhat when it came to the perceived role that practice facilitators played in implementing those benefits. Practices of both sizes viewed their facilitators as teachers, but those having 3 or fewer staff also viewed their facilitators as sources of hands-on support. ${ }^{8,21}$ This finding suggests that very small practices lack the internal capacity to implement complex systems changes and rely on their facilitators to do much of the work. Practice facilitation interventions targeting SIPs must therefore tailor their approach based on the availability of internal support staff and may experience difficulties in sustaining certain activities (eg, running EHR reports) once the facilitator leaves. Additionally, findings highlight the need to support SIPs during the implementation of complex payment reform, which clinicians identified as 2 areas where practice facilitators provided substantial hands-on support.

\section{Limitations}

Our study had several limitations. In their interviews, clinicians may have given socially desirable answers, and there may have been bias in our interview sample, as only 19 of 87 invited clinicians participated. Most invited clinicians did not respond to invitations (study investigators had no prior relationship with the sites). Additionally, the study had a relatively small sample size, particularly for the subanalysis comparing practices by office staff size. We reached thematic saturation, however, and additional interviews likely would not have yielded further themes. The qualitative interviews were also conducted in the context of a cardiovascular disease-focused trial, and the practice facilitators were employed by a single organization (the Primary Care Information Project) with a practice facilitation approach that focused on the Chronic Care Model and EHR support. Results may therefore not be generalizable to other practice facilitation interventions/programs. Lastly, all participating SIPs had a long-term relationship with the Primary Care Information Project, so results may not apply to SIPs that are new to practice facilitation or that did not enroll in HHNYC because of a poor prior experience with practice facilitation.

\section{Conclusions}

SIP clinicians derive considerable value from practice facilitation interventions; this benefit is especially evident for those with few internal office staff available to perform QI tasks. National initiatives should continue to invest in practice facilitation programs and research to support SIPs that may otherwise struggle to keep pace with the often-changing and complex health care system.

To read or post commentaries in response to this article, see it online at http://www.AnnFamMed.org/content/17/Suppl_1/S17.

Key words: primary care; practice transformation; practice facilitation; organizational change; professional practice; physician perspective; small independent primary care practices; qualitative research; health information technology; practice-based research

Submitted August 31, 2018; submitted, revised, May 20, 2019; accepted May 28, 2019.

Author contributions: D.R.S. was the study principal investigator. D.R.S., E.S.R., S.A.K., and C.A.B. codesigned and conducted the clinician interviews. E.S.R. and A.M.C. conducted all data analyses. E.S.R. led the manuscript writing. All authors contributed to data interpretation and manuscript preparation.

Funding support: This project was supported by grant number 1R18HS023922 from the Agency for Healthcare Research and Quality (AHRQ).

Disclaimer: The content is solely the responsibility of the authors and does not necessarily represent the official views of AHRQ. The corresponding author had full access to all the data in the study and had final responsibility for the decision to submit for publication.

\section{References}

1. Centers for Disease Control and Prevention. National diabetes statistics report, 2017. https://www.cdc.gov/features/diabetes-statisticreport/index.html. Accessed Dec 27, 2018.

2. Kane CK, Emmons DW. New Data On Physician Practice Arrangements. Chicago, IL: American Medical Association; 2013. https:// www.ama-assn.org/sites/ama-assn.org/files/corp/media-browser/ premium/health-policy/prp-physician-practice-arrangements_0.pdf. Accessed Dec 27, 2018. 
3. Burke T. The health information technology provisions in the American Recovery and Reinvestment Act of 2009: implications for public health policy and practice. Public Health Rep. 2010;125(1): 141-145.

4. Centers for Medicare \& Medicaid Services. Quality payment program overview. https://qpp.cms.gov/about/qpp-overview. Accessed Dec 27, 2018.

5. Marsteller JA, Woodward P, Underwood WS, Hsiao CJ, Barr MS. Design of a quality and performance improvement project for small primary care practices: reflections on the Center for Practice Innovation. Qual Prim Care. 2011;19(1):49-57.

6. Landon BE, Normand SL. Performance measurement in the small office practice: challenges and potential solutions. Ann Intern Med. 2008;148(5):353-357.

7. Baskerville NB, Liddy C, Hogg W. Systematic review and metaanalysis of practice facilitation within primary care settings. Ann Fam Med. 2012;10(1):63-74.

8. Harvey G, Loftus-Hills A, Rycroft-Malone J, et al. Getting evidence into practice: the role and function of facilitation. J Adv Nurs. 2002; 37(6):577-588.

9. Nagykaldi Z, Mold JW, Aspy CB. Practice facilitators: a review of the literature. Fam Med. 2005;37(8):581-588.

10. Berwick DM. Disseminating innovations in health care. JAMA. 2003; 289(15):1969-1975.

11. Liddy C, Singh J, Guo M, Hogg W. Physician perspectives on a tailored multifaceted primary care practice facilitation intervention for improvement of cardiovascular care. Fam Pract. 2016;33(1):89-94.

12. Shelley DR, Ogedegbe G, Anane S, et al. Testing the use of practice facilitation in a cluster randomized stepped-wedge design trial to improve adherence to cardiovascular disease prevention guidelines: HealthyHearts NYC. Implement Sci. 2016;11(1):88.
13. Wagner EH, Austin BT, Von Korff M. Organizing care for patients with chronic illness. Milbank Q. 1996;74(4):511-544.

14. Coleman K, Austin BT, Brach C, Wagner EH. Evidence on the Chronic Care Model in the new millennium. Health Aff (Millwood). 2009;28(1):75-85.

15. Kaye K, Singer J, Newton-Dame R, Shih SC. Health information technology and the primary care information project. Am J Public Health. 2014;104(3):e8-e9.

16. University at Buffalo, School of Public Health and Health Professions. Practice facilitator program. http://sphhp.buffalo.edu/home/ education/continuing-education/practice-facilitator-program.html. Accessed Dec 27, 2018.

17. Shelley D, Blechter B, Siman N, et al. Quality of cardiovascular disease care in small urban practices. Ann Fam Med. 2018;16(Suppl 1):S21-S28.

18. Damschroder LJ, Aron DC, Keith RE, Kirsh SR, Alexander JA, Lowery $J C$. Fostering implementation of health services research findings into practice: a consolidated framework for advancing implementation science. Implement Sci. 2009;4:50.

19. Hemler JR, Hall JD, Cholan RA, et al. Practice facilitator strategies for addressing electronic health record data challenges for quality improvement: EvidenceNOW. J Am Board Fam Med. 2018;31(3): 398-409.

20. Grumbach K, Mold JW. A health care cooperative extension service: transforming primary care and community health. JAMA. 2009; 301(24):2589-2591

21. Berta W, Cranley L, Dearing JW, Dogherty EJ, Squires JE, Estabrooks CA. Why (we think) facilitation works: insights from organizational learning theory. Implement Sci. 2015;10:141. 